\title{
Computed tomography and plain radiography in experimental fracture healing
}

\author{
Ethan M. Braunstein, M.D. ${ }^{1}$, Steven A. Goldstein, Ph.D. ${ }^{2}$, Janet Ku, M.S. ${ }^{2}$, Patrick Smith, M.D. ${ }^{2}$, \\ and Larry S. Matthews, M.D. ${ }^{2}$ \\ Departments of ${ }^{1}$ Radiology and ${ }^{2}$ Surgery, Section of Orthopaedics, Biomechanics Laboratory, University of Michigan, \\ Ann Arbor, Michigan, USA
}

\begin{abstract}
We evaluated the relative contribution of plain radiographs and computed tomography to the assessment of fracture healing under experimental circumstances. In 15 sheep, we performed midshaft femoral osteotomies and internal fixation of the resultant segmental fractures. Radiographs were obtained preoperatively and immediately postoperatively. Animals were sacrificed at 3 weeks, 6 weeks, 12 weeks, 24 weeks, and 36 weeks after surgery, and the femoral specimens radiographed. After removal of the internal fixation devices, computed tomographic scans of the specimens were performed.

By 3 weeks, callus was visible, but at 6 weeks, a trabecular pattern in the callus was seen on plain films but not on computed tomography. There was progressive organization of the callus on both studies. At 24 weeks, computed tomography demonstrated fracture lines not seen due to overlying callus on plain films and also more accurately showed incomplete union. By 36 weeks, healing was essentially complete according to both modalities, although there still were small gaps in the callus detectable on computed tomography but not on plain films.

Computed tomography may be of value in the evaluation of fractures of long bones in those cases in which clinical examination and plain radiographs fail to give adequate information as to the status of healing.
\end{abstract}

Key words: Computed tomography - Fracture healing

Radiologic evaluation of healing fractures and bone grafts has important implications for patient

Address reprint requests to: Ethan M. Braunstein, M.D., Department of Radiology, University of Michigan Hospitals, Ann Arbor, MI 48109, USA management, but is may be difficult to interpret such radiographs. Computed tomography (CT) in the plane axial to the shaft of a long bone may provide information about such healing unobtainable on radiographs in conventional projections. Under controlled circumstances, we examined the utility of plain radiography and CT in experimental fracture healing to see if CT offered useful additional information.

\section{Materials and methods}

In 15 adult female sheep, via a lateral approach, we resected a $4 \mathrm{~cm}$ long, mid-diaphyseal section of the femur with an osteotomy saw. The segment was autoclaved at $210^{\circ} \mathrm{C}$ for $10 \mathrm{~min}$ and then replaced in the femur in an anatomic position. Stability was assured by internal fixation with a Kuntschner nail. When normal weight bearing by the animal was observed postoperatively (approximately 3 weeks), each sheep was placed on a farm where it could continue to bear weight and to resume normal activities. Radiographs of the femur were obtained both preoperatively and immediately postoperatively.

Two animals were killed 3 weeks after surgery, and three animals each were killed at 6 weeks, 12 weeks, 24 weeks, and 36 weeks. The remaining animal was killed at 52 weeks. After killing, anteroposterior and lateral radiographs of the femur were obtained, and after the removal of the Kuntschner nails, CT was performed. We used a General Electric CT/T 9800 scanner, and exposures were made at $120 \mathrm{kVp}$ and 240 mas. The window level was 250 Hounsfield units, and the window width was 1,000 units. Beginning at the center of the fragment that had been resected and replaced, $5 \mathrm{~mm}$ thick slices were obtained from a distance of $30 \mathrm{~mm}$ proximal to this point to $30 \mathrm{~mm}$ distal to it. The radiographs and CT scans were then reviewed and compared.

\section{Results}

On radiographs immediately after surgery, a welldefined fracture line was seen without callus or resorption at each fracture site. Three weeks after fracture and internal fixation, plain radiographs of the resected femur demonstrated minimally organized callus around the site, but the osteotomy 

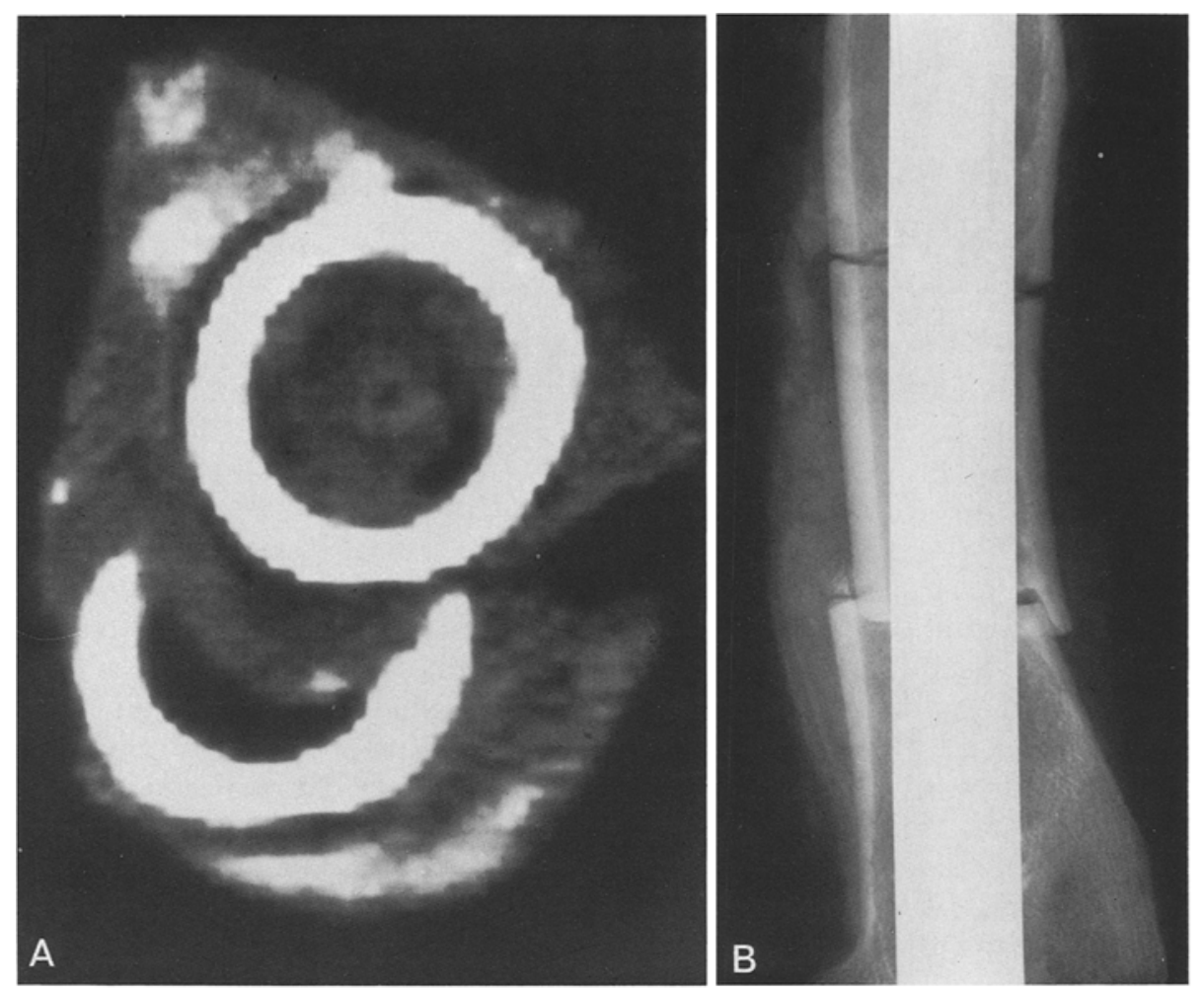

Fig. 1A, B. Three weeks after osteotomy

A On CT, the overlapping sharp edges at the osteotomy are well defined. There is a small amount of callus with no trabecular. pattern.

B Plain radiography shows sharp definition of the osteotomy sites and a small amount of poorly organized callus
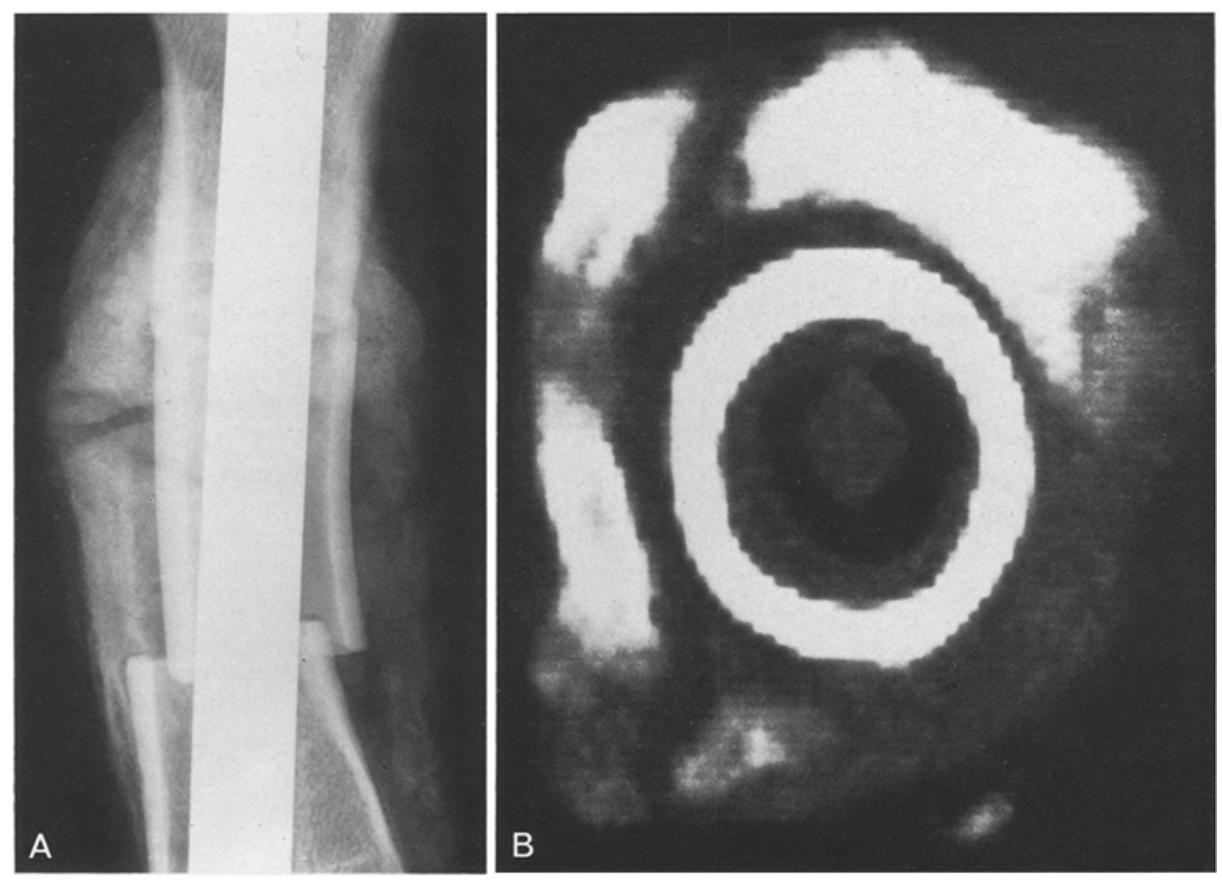

Fig. 2A, B. Six weeks postoperative.

A On plain radiographs, although the osteotomies are still well defined, there is abundant callus with the beginning of a cortical and trabecular pattern.

B On CT, callus is seen in a concentric fashion around the cortex, but organization of the callus is not appreciated as well as it is on plain radiographs

remained clearly defined without bony resorption (Fig. $1 \mathrm{~B}$ ). CT also showed clearly defined margins and a small amount of immature callus (Fig. 1A). On images obtained at 3 weeks, information obtained by the two techniques was comparable.

In the 6-week specimens, the osteotomy was still well defined on plain radiographs (Fig. 2A). By this time, there was progressive maturity of the external callus with an organized trabecular and cortical pattern. On $\mathrm{CT}$ in the axial projection, the concentric nature of the external callus could be appreciated better than on plain radiographs, 

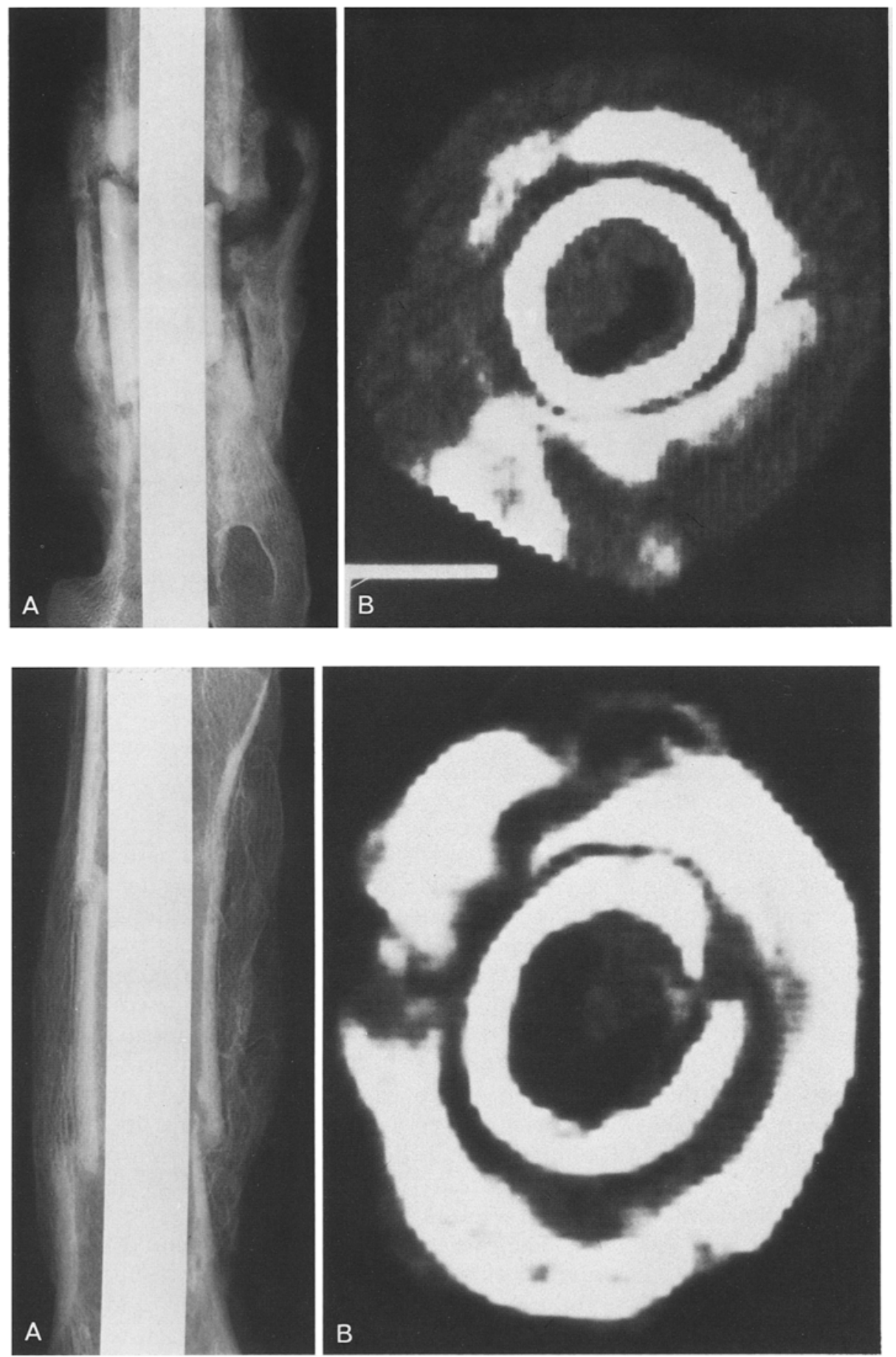

Fig. 3A, B. Twelve weeks postoperative.

A Plain radiographs show organized callus bridging the osteotomy sites. Notice the mature trabecular bone in the callus.

B The trabecular pattern of the callus is still not well defined, and it appears less mature than on corresponding plain radiographs

Fig. 4A, B. Twenty four weeks postoperative. A Plain radiographs show apparent bony union with mature bridging callus.

B On CT, the concentric callus is shown to mask a cortical gap. Healing is not as complete as it appears on plain radiographs

but CT did not define the internal trabecular structure of the callus (Fig. 2B).

At 12 weeks, on plain radiographs, definite bridging of the external callus across the fracture site was demonstrated, and the trabecular nature of this new bone was clearly seen. The edges of the fracture were not as clearly seen as they had been on earlier specimens, presumably indicating resorption and healing (Fig. $3 \mathrm{~A}$ ). On CT, although some early cortex was formed in the external callus, the trabecular pattern was not as clearly defined as it was on the plain radiographs. Indeed, a cloud-like pattern of immature callus still predominated. The fracture lines remained well defined with no further evidence of union (Fig. 3B). 

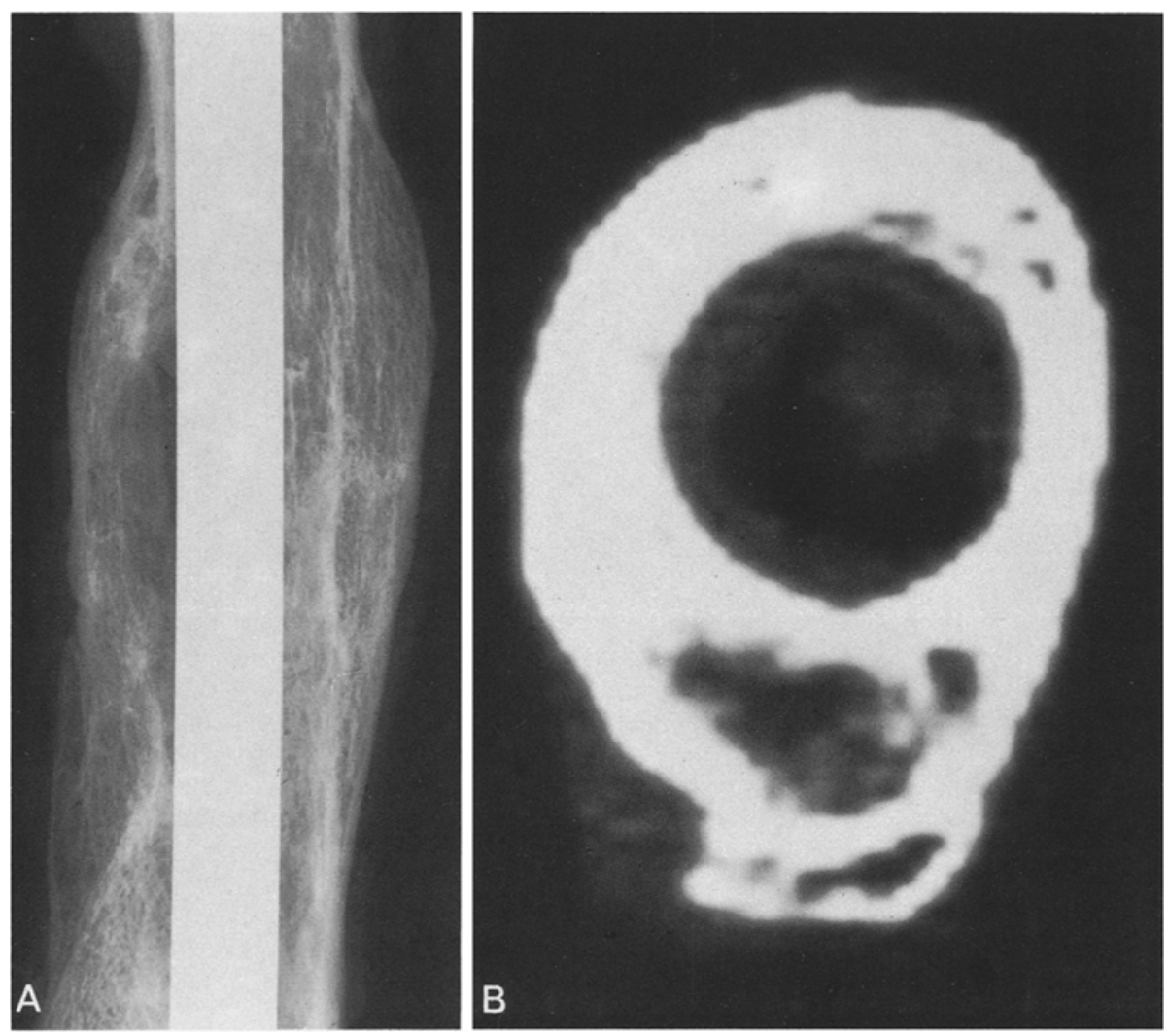

Fig. 5A, B. Thirty six weeks postoperative.

A Plain radiographs demonstrate complete bony union with reincorporation of the intercalated bony segment into the femoral shaft.

B CT also demonstrates bony union

By 24 weeks, there had been apparent bony union of the fracture on plain radiographs, as the fracture lines were obliterated. There also had been further remodelling of the external callus into a bony "envelope" surrounding the osteotomy site (Fig. 4A). However, on CT the apparent union is shown to be due to the overlap of the concentric external callus, and there continues to be slight separation of the cortical fragments of the shaft (Fig. 4B). A some levels there is bony union, but the process is not as complete as plain radiographs suggest.

Finally, at 36 weeks there is complete union seen on plain radiographs, with obliteration of the fracture sites. There has been total reincorporation of the grafted segment into the femoral shaft (Fig. 5A). On CT, there also is mature bridging external callus at most levels from $30 \mathrm{~mm}$ proximal to the midpoint of the grafted segment to $30 \mathrm{~mm}$ distal to the midpoint (Fig. 5B). In a few isolated areas, there are gaps in the external callus, but the process of bony union is essentially complete.

\section{Discussion}

Although the physiologic stimulus for fracture and bone graft healing is poorly understood, such heal- ing occurs in well-defined histologic patterns which have radiologic correlates. Immediately after a fracture or osteotomy, there is bleeding from the bone ends and from surrounding soft tissue. After a clot forms, fibroblasts begin to penetrate this hematoma, which undergoes fibro-osseous metaplasia [2]. Early periosteal callus begins to surround the fracture site, and collagen forms as fibroblasts undergo metaplasia to chondroblasts by the end of the first week. Although there may be blurring of the fracture line by this time [5], in our study the osteotomy margins remained evident more than 3 weeks after surgery.

The earliest organizing callus should be visible two to three weeks postoperatively, and this was the case both on plain radiographs and on CT. This external callus bridges the fracture site. As it organizes, a trabecular pattern emerges as osteoid is formed from the enchondral bone produced in the callus. This pattern appeared earlier on plain radiographs than on CT, perhaps due to better spatial resolution on the former.

By 12 weeks, there is abundant callus, and fracture margins have become quite indistinct on plain radiographs. Finally, on our 24 and 36 week specimens, there has been complete bony bridging, as medullary as well as external callus has formed; 
and there has been direct osteonal penetration across the fracture gap [1]. Even at 36 weeks, there appears to be some slight cortical separation at the fracture site on CT not apparent on plain radiographs, but healing is essentially complete.

In this study, we segmentally fractured the femurs, as segmental fractures are more likely than simple linear fractures to have complications such as nonunion. Thus, we were able to reproduce more closely the conditions of severe, comminuted fractures with devascularized fragments of bone. Also, the segmental fractures closely resembled bone grafts, particularly those grafts used in reconstructive tumor surgery.

Similarly, autoclaving the segments reproduced a situation in which healing may be retarded. The autoclaved segment, when returned to the host, contained no living cells and could not contribute to the healing process. Its function was to act as a scaffold for healing via cellular ingrowth from the remainder of the femur. In many comminuted fractures, such fragments, while not autoclaved, are certainly devascularized and contain no living cells, yet they are used in anatomic reconstruction of the limb. Autoclaved bone also provides another model in clinical fracture treatment, as comminuted fracture fragments may be autoclaved, if they have been contaminated by the environment, to sterilize them before such reconstruction. In tumor surgery as well, autoclaved bone provides a clinical model. In central chondrosarcoma of long bones, the tumor-containing specimen may be resected, the tumor removed, and the remaining specimens autoclaved and replaced. This provides a structural homograft for reconstruction [4].

By radiographic criteria, our specimens did heal normally and appeared intact. Autoclaving did not seem to structurally damage the fragments, which then provided support for callus formation and osteonal penetration from the remainder of the femur.
Clinical and radiographic assessment of fracture and graft healing is difficult. Radiographic criteria include loss of the fracture line, trabeculae crossing the site of the fracture, visible callus, and bony bridging. In a controlled study [3], both radiologists and orthopedists were unable to detect early bony union, and none of the observers could determine which specimens had the strongest union. From our study, plain radiography and CT appear to be complementary in the evaluation of fracture and bone graft healing. The better spatial resolution of plain radiographs allows earlier detection of external callus, but CT may show lack of primary union even in the presence of external callus. This is particularly true when the callus surrounds the fractured shaft, obscuring the remaining fracture line. Of course, in the clinical setting the usefulness of CT is limited if there is internal fracture fixation by metallic devices. CT may offer information differing from that on plain radiographs. Thus, in selected cases, CT may be of benefit when clinical and radiographic examination differ in the assessment of fracture healing.

Acknowledgements. Portions of this work were supported by the University of Michigan Cancer Research Institute and the Department of Surgery, University of Michigan.

\section{References}

1. McKibbin B (1978) The biology of fracture healing in long bones. J Bone Joint Surg [Br] 60:150

2. Naimark A, Miller K, Segal D, Kossoff J (1981) Nonunion. Skeletal Radiol 6:21

3. Nichols PJ, Berg E, Bliven FE, Kling M (1979) X-ray diagnosis of healing fractures in rabbits. Clin Orthop 142:234

4. Smith WS, Simon MA (1975) Segmental resection for chondrosarcoma. J Bone Joint Surg [Am] 57:1097

5. Weissman BN (1983) Fracture healing. In: Syllabus for the categorical course on musculoskeletal trauma, presented at the annual meeting of the American Roentgen Ray Society, Atlanta. American Roentgen Ray Society, Baltimore, p 37 